\title{
STARSPOTS ON BY DRA-TYPE STARS
}

\author{
D.S. EVANS \\ University of Texas, Austin, Texas
}

The first detection of spots on dwarf M stars was by Kron on YY Geminorum in 1948. Since then phenomena attributable to starspots have been found by numerous authors beginning with Kraft and Krzeminski on the star now known as BY Draconis and on CC Eridani by Evans. There is a close correlation between spottedness, Balmer emission and flaring in dwari li stars.Evans appears to have been the first to attempt to analyse the small range light variations on spotted stars in terms of modulation caused by axial rotation. In comparison with the solar case one must assume that the spots are very large and long lived. There is also an ambiguity in dealing with very large dark spots as between a star with a dark area or a cool star with a bright area (the zebra effect-is a zebra a black animal with white stripes or a white animal with black stripes?). It is difficult to arrive at a solution for the spot parameters which is unique. In particular several analyses which have appeared heve assumed that maximum light corresponds to the immaculate star whereas in fact the maximum light in the variation corresponds to the immaculate star dimmed by the light of any constant star abstraction.

In the case of BY Draconis for which the orbit of Bopp and Evans gives an inclination of $30^{\circ}$ approximately it seems possible to arrive at a definitive solution. In a paper by Oskanyan, Evans, Lacy and McMillan a long series of photometric observations made at Byurakan Observatory in Soriet. Armenia and at McDonald is analysed. These can all be represented by small range sinusoidal variations and the following plausible assumptions can be made. (a) That since the light curve shows no level portion the spot lies wholly in the polar area always exposed to view and that no part of it lies in the equatorial zone where it would sometimes be exposed and sometimes hidden at each revolution. (b) That a sinusoidal variation through a range of a few hundreths of a magnitude is most economically explained as due to a single active area. The data string is best represented by a series of different sinusoidal trains with parameter changes between trains. A simple mathematical treatment relates amplitude of variation to

Edith A. Müller (ed.), Highlights of Astronomy, Vol. 4, Part II, 395-396. All Rights Reserved. Copyright (C) 1977 by the IAU. 
depression of the mean level as a function of latitude of the spot. The results depend on the assumption that all the spots observed have been on the brighter component (only a single periodicity has been found! but are not dependent on the assumed luminosity ratio between the components. We infer that spots occur in high latitudes, even in the extreme polar recion. The phase jump of 2.1 drys found by Krzeminski (approximately half the rotation period of 3.9 days) is then readily explained as due to migration of the spot over the polar region. The spot has migrated both in latitude and longitude and there is no evidence of a systematic change of period with latitude. Consideration of 011 data covering the last 20 years suggests that a large dark spot. appeared in 1965, migrated over the pole in 1968, and was replaced by a bright area in 1970 (when the value of $B-V$ showed a reduction). This bright area is not due to l.ine emission which in recent years has been weak. 